\title{
Intermediate plasmonic characteristics in a quasi-continuous metallic monolayer
}

SUBJECT AREAS:

NANOPHOTONICS AND

PLASMONICS

NANOPARTICLES

Received

2 August 2013

Accepted

16 December 2013

Published

15 January 2014

Correspondence and requests for materials should be addressed to B.L. (byoungho@snu. ac.kr)

\author{
Hansik Yun' ${ }^{1}$ II-Min Lee', Seung-Yeol Lee', Kyoung-Youm Kim² \& Byoungho Lee'
} 1National Creative Research Center for Active Plasmonics Application Systems, Inter-University Semiconductor Research Center and
School of Electrical Engineering, Seoul National University, Seoul 151-744, Republic of Korea, ${ }^{2}$ Department of Optical Engineering,
Sejong University, Seoul 143-747, Republic of Korea.

There has been a significant interest on plasmonics in a metallic structure with very narrow gaps for studies of nanophotonics. However, little attention has been paid to the behavior of surface plasmons (SPs) in quasi-continuous metallic structures. This study observes and analyzes intermediate characteristics between propagating SPs (PSPs) and localized SPs (LSPs) in a quasi-continuous metallic monolayer of core-shell nanocubes. We reveal that, in a very narrow region of few-nanometer gaps among the nanocubes, the intrinsic energy bands of PSPs and LSPs intersect each other to generate two hybrid bands and an anti-crossing. Using a self-assembly method instead of the lithographic techniques which have several limitations as of now, we materialize the quasi-continuous metallic layer with plenty of nano-gaps that exhibit intermediate plasmonic characteristics. The intermediate plasmonic characteristics observed in this study will lead to interesting subjects, such as band engineering and slow SPs, in nanophotonics.

$\mathrm{n}$ macroscopic electromagnetism, a material with negligible discontinuities can be considered as a continuous medium if the length scales of the discontinuities are far below the wavelength. In the surface plasmons (SPs), defined as the collective oscillations of free electrons stimulated by light at an interface between metal and dielectric, however, the field can be significantly influenced by very narrow discontinuities which are as small as one ten-thousandth of the wavelength ${ }^{1-3}$. Therefore, in plasmonics, more stringent criteria are required for a continuous medium. To be more specific, it is expected that the continuity of a metallic structure has a relation with the dominant state of SPs in that structure. As is well-known, there are two independent states in surface plasmons (SPs): propagating SPs (PSPs) and localized SPs (LSPs). PSPs propagate along the smooth and continuous surface of a metal layer ${ }^{4-6}$, while LSPs reside around a small or distinct metallic nanostructure ${ }^{7-10}$.

Based on this perspective, our attention is naturally focused on the discontinuity-related intermediate characteristics of SPs. By intuition, it is expected that a transition of SPs from LSPs to PSPs will be observed if the sizes of the discontinuities in a metallic layer are reduced from significant to negligible lengths and eventually to zero, and vice versa. Since the light fields associated with SPs can be confined in a small inclusion of discontinuity of a metallic structure, such transition between the PSPs and LSPs may occur for very narrow discontinuities, of which the size is far below the diffraction limit. Although there have been several studies about the coupling between LSPs and PSPs, including the periodic metal particles coupled to a separated metal film ${ }^{11-13}$ or metallic holes ${ }^{14-16}$, they did not consider the transition behavior of plasmonic states in an almost-continuous metallic layer. In respect of the almost-continuous structure, some research groups have introduced a metal-insulator transition ${ }^{17}$ and tunable plasmonic property ${ }^{18}$ at close-packed metal nanoparticles. While the previous researches broadly suggest the potential of a bottom-up strategy and their properties, this study can provide a detailed map of unique dispersion bands of SPs in one structure and concretely demonstrate their intermediate behaviors depending on structural parameters, as well as an in-depth understanding of the boundaries between the states of the SPs.

This study intends to investigate the states of SPs in a metallic layer with very narrow discontinuities. The sizes of the discontinuities considered in this study are ranged from zero to a few nanometers. As one of the feasible implementations for the said structure, we propose a monolayer of metal nanocubes with thin dielectric shells. Also, a bottom-up approach is used, instead of the top-down process, such as lithography which is extremely difficult to apply, so as to build a self-assembled metallic monolayer with nano-sized discontinuities. Nanodiscontinuities can be materialized with few-nanometer-thin dielectric shells among the neatly spaced metal nanocubes. Hereafter, we refer to the monolayer of nanocubes in this study as "a quasi-continuous metallic layer (QCML)". From the simulations and experiments of reflectometry, it is found that two distinct energy bands originating from the PSPs and LSPs can be observed with a phenomenon of anti-crossing in QCML. Each of the two bands exhibits intermediate natures of PSPs and LSPs due to their strong coupling. The dispersive nature of 
QCML reveals the evolution or transition mechanism of the LSPs in the isolated metallic structure into PSPs in the continuous structure. In addition, the QCML provides a novel way of tailoring the dispersion characteristics of the SPs or generating slow SPs by adjusting the size of discontinuities.

\section{Results}

A concept of a monolayer of core-shell nanocubes for a quasicontinuous metallic layer. Let us consider a monolayer of tightlyspaced metal nanocubes as shown in Fig. 1(a). Throughout this study, we use the silver nanocubes $(\mathrm{AgNC})$ with silica $\left(\mathrm{SiO}_{2}\right)$ shells $\left(\mathrm{AgNC} @ \mathrm{SiO}_{2} \mathrm{~s}\right)$ as the elements of QCML. If the shell-thickness of cubes is zero, the structure can be considered as a continuous metal layer so that PSPs can be excited via the Kretschmann configuration $^{4-6}$. If the cubes are largely separated with thick shells, the layer can be assumed as a monolayer of particle array so that only the isolated LSPs can be excited ${ }^{7-10}$. Meanwhile, when the distances among the metal cubes are in a range from zero to the distances enough to isolate the cubes, intermediate plasmonic characteristics can be found between PSPs and LSPs due to their strong coupling in the QCML. For the structures with different inter-particle distances, we examined the dispersive characteristics of SPs via angle-resolved spectral reflectometry, as shown in Fig. 1(b). Since the incident angles under consideration are in the total internal reflection (TIR) regime, the minima in the reflection curve are corresponding to the excitation of SP modes ${ }^{4-6}$.

Intermediate plasmonic characteristics and anti-crossing. In this study, the results were produced using a Fourier-modal analysis based numerical method ${ }^{19-22}$. In order to make the process of calculation simple and efficient, we performed two-dimensional (2D) simulations instead of three-dimensional (3D). For the AgNC@SiO $\mathrm{S}_{2}$ monolayer consisting of $35 \mathrm{~nm}$ AgNC core and $2 \mathrm{~nm}$ $\mathrm{SiO}_{2}$ shell, by varying the incident wavelengths from $400 \mathrm{~nm}$ to $800 \mathrm{~nm}$ under the incident angles from $40^{\circ}$ to $80^{\circ}$, the dispersion characteristic $\left(\theta-\lambda_{R}\right.$ map) was obtained as shown in Fig. 1(c).

Interestingly, while only one intrinsic band for PSPs or LSPs was observed in a continuous case and an isolated case (insets of Fig. 1(a)), the two bands of SPs appeared when the shell thickness was in the intermediate range between the two extreme cases. In the conventional energy-momentum $(E-k)$ diagram in Fig. $1(\mathrm{~d})$ that is clockwisely rotated by $90^{\circ}$ from the $\theta-\lambda_{R}$ map in Fig. 1(c), the extrapolated bands (the red and blue dotted lines) of PSPs and LSPs accord with the traditional $P$ - and $L$-bands in Figs. 1(e) and (f). Since PSPs can be excited via the Kretschmann configuration using the prism, the $L$-band can be particularly separated from the two bands in Fig. 1(c) by the removal of the prism under the QCML, as shown in Fig. 1(f). Further details about $P$ - and $L$-bands are explained in Fig. S1 of the Supplementary Information. In this QCML, therefore, the two distinct plasmonic modes of PSPs and LSPs coexist in the same structure, and $P$ - and $L$-bands cross each other. Interestingly enough, at the supposed crossing point of these two bands, an anti-crossing phenomenon, which is a manifestation of strong coupling of two modes, is observed.

To obtain further physical insights for the intermediate characteristics and the anti-crossing, we examined $H_{y}$-field profiles at six distinguishing points as shown in Fig. 1(c). In Figs. 1(g)-(l), the TM-polarized planewave of light is obliquely incident onto the metallic layer near $z=0$ from the downside. In this figure, the supposed crossing point of two intrinsic bands is depicted as ' $O$ ' in Figs. 1(c) and (d). When the excitation conditions are far from the ' $O$ ' point, the field profiles of the SPs in the intermediate structure resemble those of the intrinsic PSP (Figs. 3(g) and (l)) and LSP (Figs. 3(i) and (j)) modes. However, near the ' $O$ ' point (Figs. 3(h) and (k)), the field profiles for the upper (left in Fig. 1(c)) and the lower (right in Fig. 1(c)) energy bands in Fig. 1(d) exhibit hybridized modes of PSPs and LSPs. In Fig. 1(h), the fields for the LSPs in the metallic layer are in anti-phase with those for the PSPs on the surface. On the contrary, PSPs and LSPs are in-phase in Fig. 3(k). As a consequence, the anti-phase hybrid modes of PSP and LSP are in the higher (left in Fig. 1(c)) energy band while the in-phase modes are in the lower (right in Fig. 1(c)) energy band. Such hybridizations of modes and corresponding energy relations are similar to those that can be found in the hybrid modes of coupled waveguides ${ }^{23-26}$.

In brief, PSPs and LSPs coexisted in our QCML and anti-crossing was observed as an evidence of the strong coupling of these two types of SPs, which indicates that the PSPs and LSPs consist of the eigenstates in our plasmonic structure. In addition, in this study, we chose water (refractive index 1.33) as the surrounding material because the behavior of the bands is well observed under that condition. The $\theta-\lambda_{R}$ maps of the structure with air as the surrounding material are shown in Fig. S2 of the Supplementary Information.

Effects of core size and shell thickness. It is important to investigate the intermediate range and the behavior of the two hybrid bands according to the structural parameters including $\mathrm{SiO}_{2}$ shell thickness and AgNC core size. First, in Fig. 2(a), the increment of shell thickness induces a blue-shift in the location (energy level) of the $L$-band. It is because the inter-particle distance which is determined by shell thickness decides the resonance wavelength of the $\operatorname{LSPs}^{27-29}$. In this case (35 $\mathrm{nm}$ of AgNC core), the intermediate characteristics by the strong coupling between PSPs and LSPs can be observed in a very narrow region where the shell thickness is below $8 \mathrm{~nm}$, because $P$ - and $L$-bands start to overlap at that thickness.

The behavior of the bands is also affected by the size of the metal core as in Fig. 2(b): the core size determines mainly the relative strength of the PSP and LSP modes in their coupling. As the core size gets larger, the excitation of the PSPs gets weaker since the launching of PSPs with the Kretschmann configuration through a thick metal becomes harder. Therefore, the depth of the reflection dip for the PSP mode ( $P$-band) in Fig. 2(b) gets weaker with the increment of the core size. On the contrary, the excitation of the LSPs gets weaker with the decrement of the core size. In Fig. 2(b), the $L$-band abates rapidly as the core size gets smaller because the scattering and absorption intensities of particles generally are proportional to the six and third power of a particle size, respectively ${ }^{30,31}$.

In brief, as the sizes of dielectric shell and metal core increase, LSP mode becomes more dominant than PSP mode in the intermediate state, and vice versa. The behavior of each constituent part of two hybrid bands properly follows each fundamental principle of PSP and LSP, and that is an obvious evidence for the strong coupling between PSPs and LSPs. In addition, it is noteworthy that the two hybrid bands can be readily designed by engineering the geometrical parameters.

Experimental $\theta-\lambda_{R}$ map of a self-assembled monolayer of AgNC@ $\mathrm{SiO}_{2}$ s. According to the simulation results, in order to ascertain experimentally the existence of the two bands in a $\theta-\lambda_{R}$ map, metal nanocubes should have a few nanometers of narrow gaps among them. However, carving straight lines with a few nanometers in width on the metal layer is almost infeasible because the minimum line width of current lithography techniques is approximately $20 \mathrm{~nm}^{32,33}$. Alternatively, we constructed the proposed structure with metallic nanocubes because the bottom-up method can manufacture materials in a few nanometer scales ${ }^{34-37}$. Cubic structures have a geometric advantage in forming a monolayer: the cubes can contact with each other or with the substrate face-to-face so that they can be assembled denser than other shaped structures even if they are randomly scattered on the substrate. They were synthesized with the polyol method ${ }^{38,39}$ and enclosed with $\mathrm{SiO}_{2}$ shell in the Stöber process ${ }^{40,41}$. A monolayer of $\mathrm{AgNC@SiO}_{2} \mathrm{~s}$ was prepared by the self-assembled monolayer (SAM) method, which uses differences between solubility and surface tension of water 

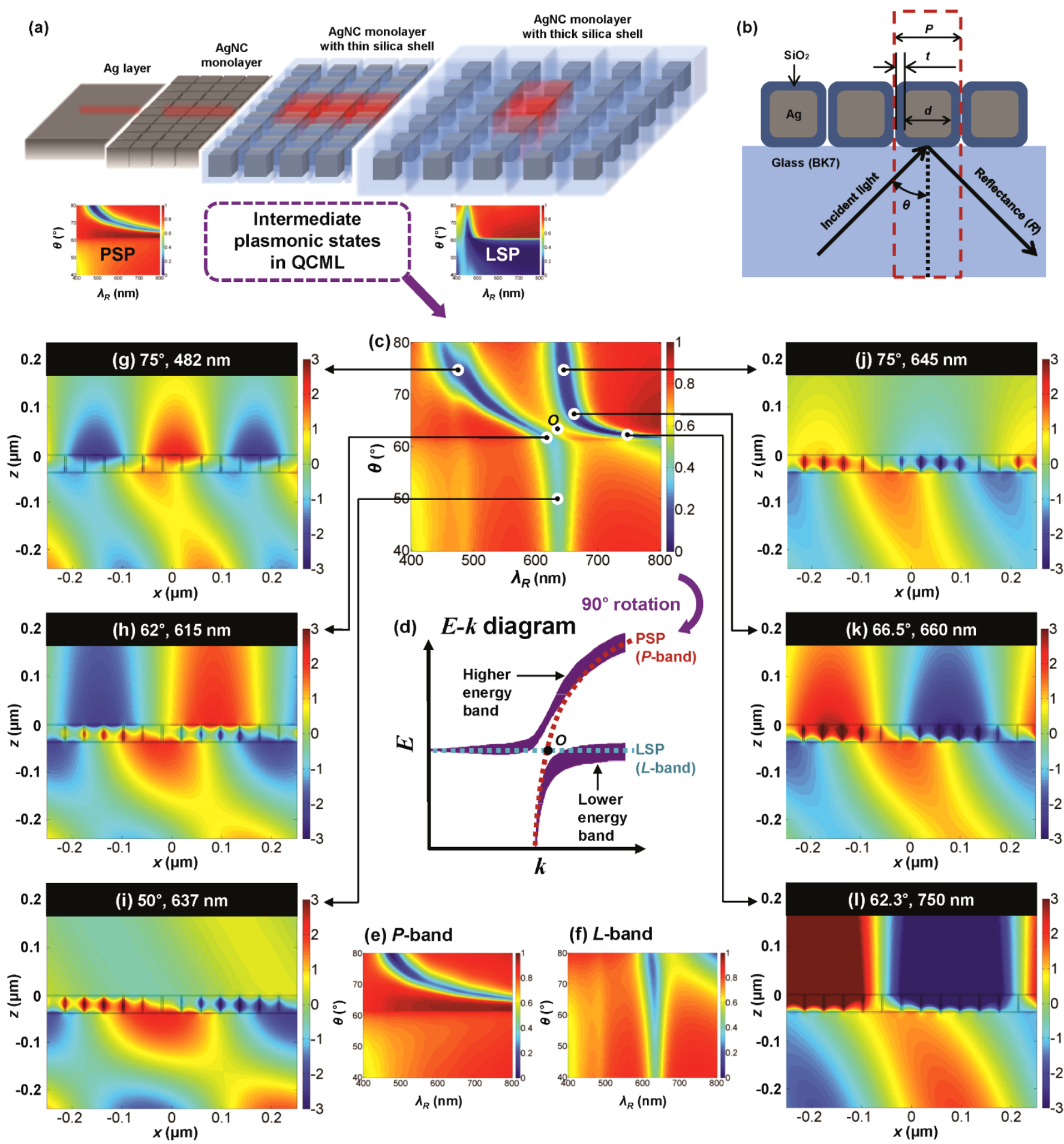

Figure $1 \mid$ Intermediate plasmonic state in QCML. (a) Concept structures for the strong coupling between PSP and LSP in a monolayer of metallic cubes. Insets show the traditional $\theta-\lambda_{R}$ maps of PSP and LSP, respectively. (b) Simulation condition. One period includes a core-shell cube. $P, d, t, \theta$, and $R$ are period, core diameter, shell thickness, incident angle, and reflectance, respectively. (c) $\theta-\lambda_{R}$ map of AgNC ( $\left.35 \mathrm{~nm}\right)$ with shell (2 nm). (d) $E-k$ diagram expressed by $90^{\circ}$ rotation of the $\theta-\lambda_{R}$ map. (e) $P$-band. (f) $L$-band. $H_{y}$-field profiles at $\theta$ and $\lambda_{R}$ of (g) $75^{\circ}$ and $482 \mathrm{~nm}$, (h) $62^{\circ}$ and $615 \mathrm{~nm},(\mathrm{i}) 50^{\circ}$ and $637 \mathrm{~nm},(\mathrm{j}) 75^{\circ}$ and $645 \mathrm{~nm},(\mathrm{k}) 66.5^{\circ}$ and $660 \mathrm{~nm}$, and (l) $62.3^{\circ}$ and $750 \mathrm{~nm}$. They show (g)-(i) transition from PSP to LSP and (j)-(l) transition from LSP to PSP.

and organic solvent where $\mathrm{AgNC@SiO} \mathrm{S}_{2}$ are dispersed ${ }^{42-44}$. As shown in Figs. 3(a) and (b), AgNC@SiO ${ }_{2}$ has approximately $43 \mathrm{~nm}$ of core size and $3.5 \mathrm{~nm}$ of shell thickness in the high resolution transmission electron microscope (HR-TEM) image and they are settled into a monolayer with high population density in the field emission scanning electron microscope (FE-SEM) image. AgNC orientations were measured from the FE-SEM image because nanocube orientations, including face-to-face, face-to-edge, and edge-to-edge orientations, have an effect on the plasmonic property ${ }^{45}$. As a result, we can confirm that more than $75 \%$ of the nanocubes contact face-to-face with each other, as shown in Fig. S3 of the Supplementary Information, and that the AgNC orientations of the experimental samples may be significantly in accord with the face-to-face orientation of the simulation. Figure 3(c) shows an illustration of the microscope setup 
(a) Core size : $35 \mathrm{~nm}$

Shell thickness : $1 \mathrm{~nm}$

$1.5 \mathrm{~nm}$

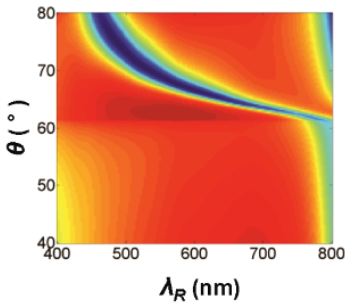

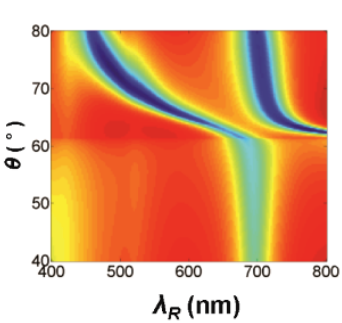
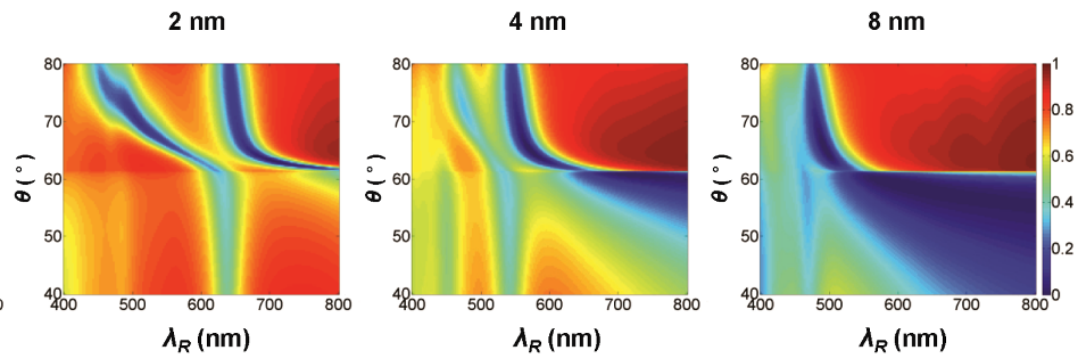

Propagating SPs

Intermediate plasmonic states

Localized SPs

(b) Shell thickness : $2 \mathrm{~nm}$

Core size : $25 \mathrm{~nm}$

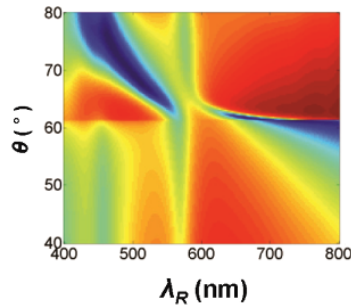

$30 \mathrm{~nm}$

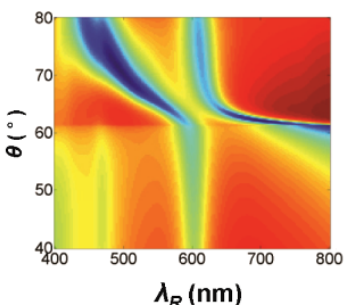

$35 \mathrm{~nm}$



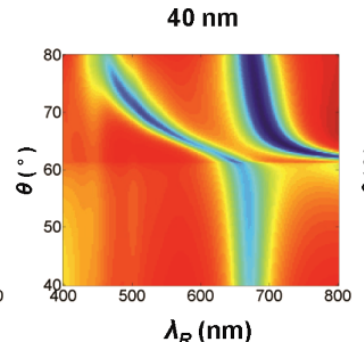

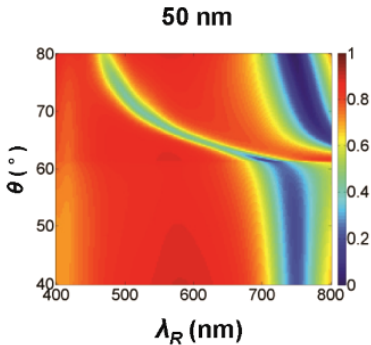

Figure $2 \mid$ Discontinuity-dependent behavior of two hybrid bands. Effect of (a) $\mathrm{SiO}_{2}$ shell thickness and (b) AgNC core size on two hybrid bands of $\theta$ - $\lambda_{R}$ map.

in order to measure an incident angle-dependent reflectance spectrum. The spectral reflectance of the sample was obtained with a spectrometer at several incident angles using a white light source in the Kretschmann configuration.

Three representative cases of experimental $\theta-\lambda_{R}$ maps are shown in Fig. 4 with the corresponding simulation $\theta-\lambda_{R}$ maps. The three samples are a continuous Ag layer (thickness $43 \mathrm{~nm}$ ) evaporated on glass in Figs. 4(a) and (d), AgNC@SiO 2 SAM (core size 43 nm, shell thickness $3.5 \mathrm{~nm}$ ) on glass in Figs. 4(b) and (e), and $\mathrm{AgNC@SiO}_{2}$ randomly scattered on glass with various broad gaps in Figs. 4(c) and (f), respectively. They were measured for water (refractive index 1.33) as surrounding medium and the corresponding results for air

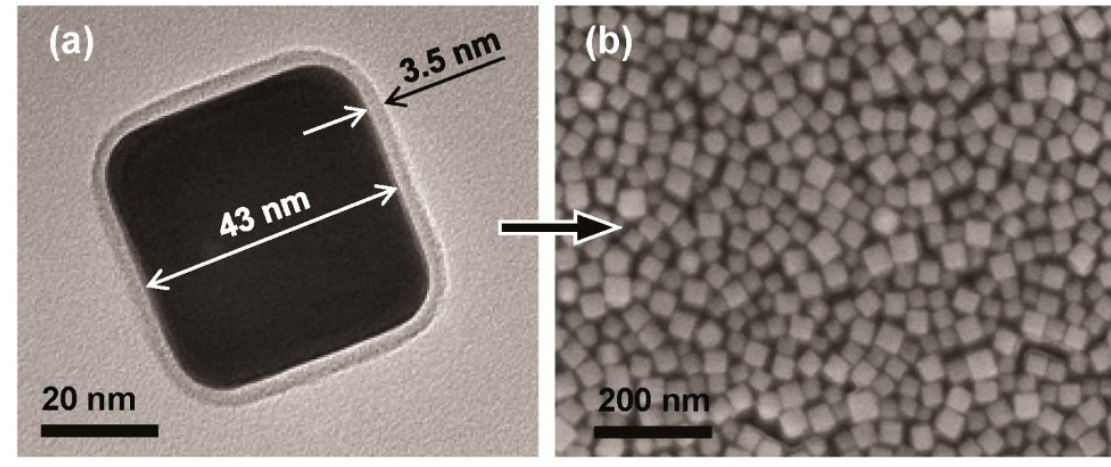

(c)

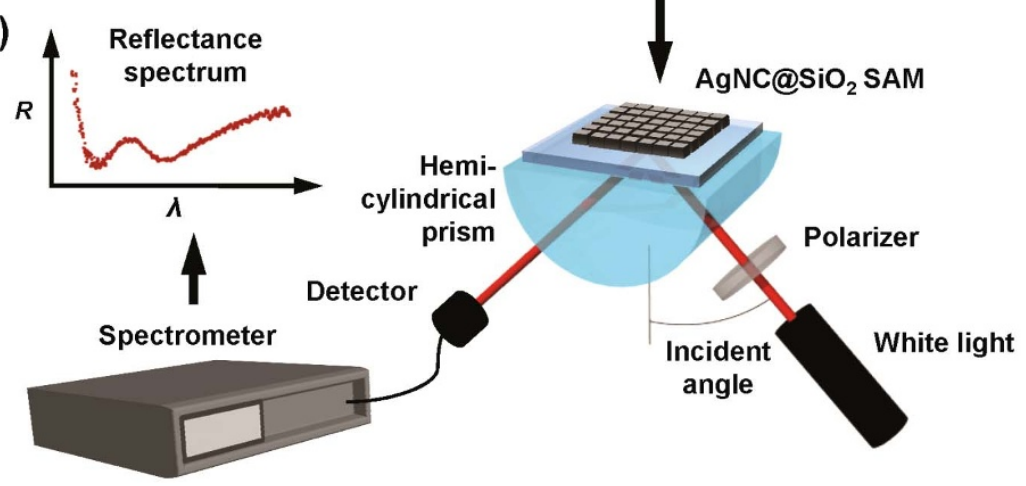

Figure 3 Experimental setup for SP excitation in $\mathrm{AgNC}_{\mathrm{S}} \mathrm{SiO}_{2} \mathrm{SAM}$. (a) TEM image of $\mathrm{AgNC}_{2} \mathrm{SiO}_{2}$. (b) SEM image of AgNC@SiO 2 SAM. (c) Schematic diagram of optical setup for measurement of incident angle-dependent reflectance spectra. Scale bars are (a) $20 \mathrm{~nm}$ and (b) $200 \mathrm{~nm}$. 
(refractive index 1.00) are shown in Fig. S4 of the Supplementary Information. The spectral reflectance data are accumulated into an experimental $\theta-\lambda_{R}$ map by scanning the incident angles between $60^{\circ}$ and $75^{\circ}$ at an interval of $1^{\circ}$. A series of the minimum reflectance dips in the experimental graphs can be regarded as a quasi-band since they shift with a specific tendency in wavelength. They are marked with round black dots on the corresponding positions in the simulation $\theta-\lambda_{R}$ maps in Figs. 4(d)-(f) to compare with each other. The single quasi-bands almost accord with $P$-band due to PSPs, and with $L$-band due to LSPs in Figs. 4(d) and (f), respectively. On the contrary, two dips appear in one spectrum and two quasi-bands emerge on the experimental $\theta-\lambda_{R}$ map in Fig. 4(b). As the incident angle increases, one quasi-band near $460 \mathrm{~nm}$ moves toward short wavelength while the other quasi-band near $600 \mathrm{~nm}$ keeps its position. The experimental $\theta-\lambda_{R}$ map in Fig. 4(b) can be the evidence for the existence of the strong coupling between PSPs and LSPs because the number and behavior of the quasi-bands are similar to the calculated $\theta-\lambda_{R}$ map in Fig. 4(e).

Statistical feedback of spatial distribution of $\mathrm{AgNC@SiO}_{2} \mathrm{~s}$ to simulation. We can reduce the differences between the experimental and calculated $\theta-\lambda_{R}$ maps by feeding back statistical data for a gap distribution of AgNC@SiO ${ }_{2}$ SAM to the prior simulation. It cannot be helped that a SAM of nanocubes has a certain distribution of gaps between them in a real sample while the nanocubes can be set at exact lattice points with the same distance of gap in a simulation. The limitation of the self-assembly method brings about differences in distance among the cubes: for example, two bands of Fig. 4(b) are placed at a shorter wavelength than those of Fig. 4(e) and the slope of the left band is different between Figs. 4(b) and (e). In order to solve the problem, we measured the inter-particle distances from the FESEM image of Fig. 3(b), as shown in Fig. 5(a). The average interparticle distance was $49.3 \mathrm{~nm}$ and the standard deviation was 7.47 nm. One hundred random numbers were generated in a normal distribution with the average and standard deviation as a distribution chart shown in Fig. 5(b), and then inputted in one period as the schematic diagram in Fig. 5(c). Namely, the calculation was done for one hundred nanocubes randomly and normally distributed in one period.

The recalculated $\theta-\lambda_{R}$ map is demonstrated in Fig. 5(f) along with the initially calculated map in Fig. 5(d) and the experimental map in Fig. 5(e) for comparison. The corresponding results for air (refractive (a)

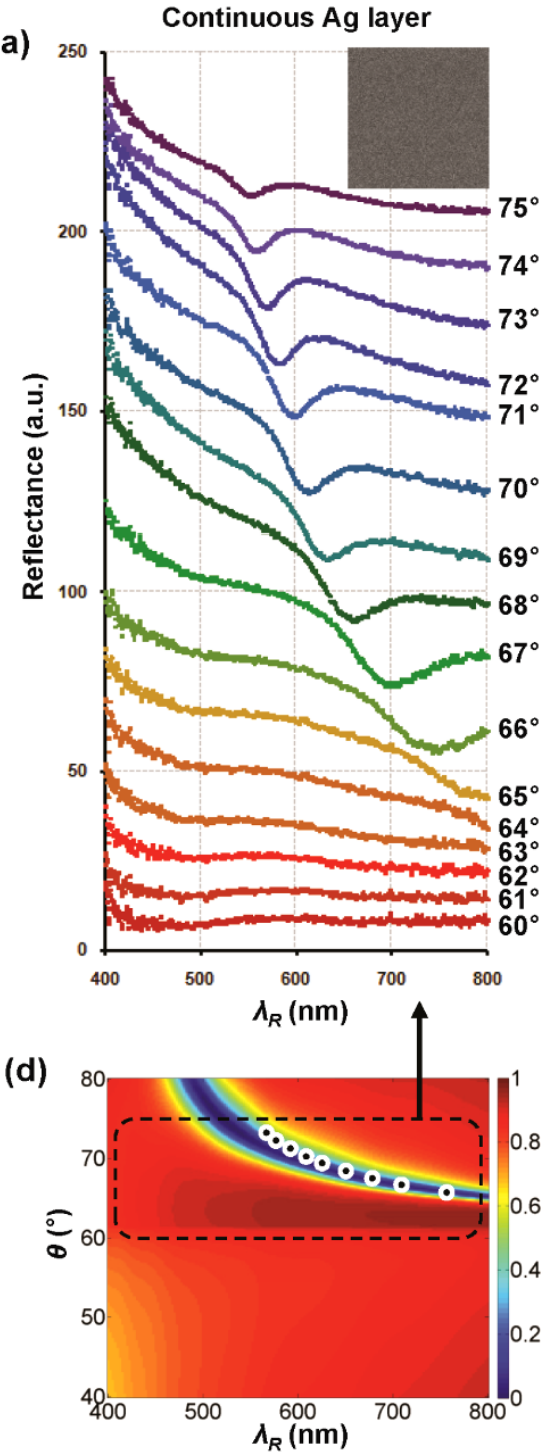

(b)

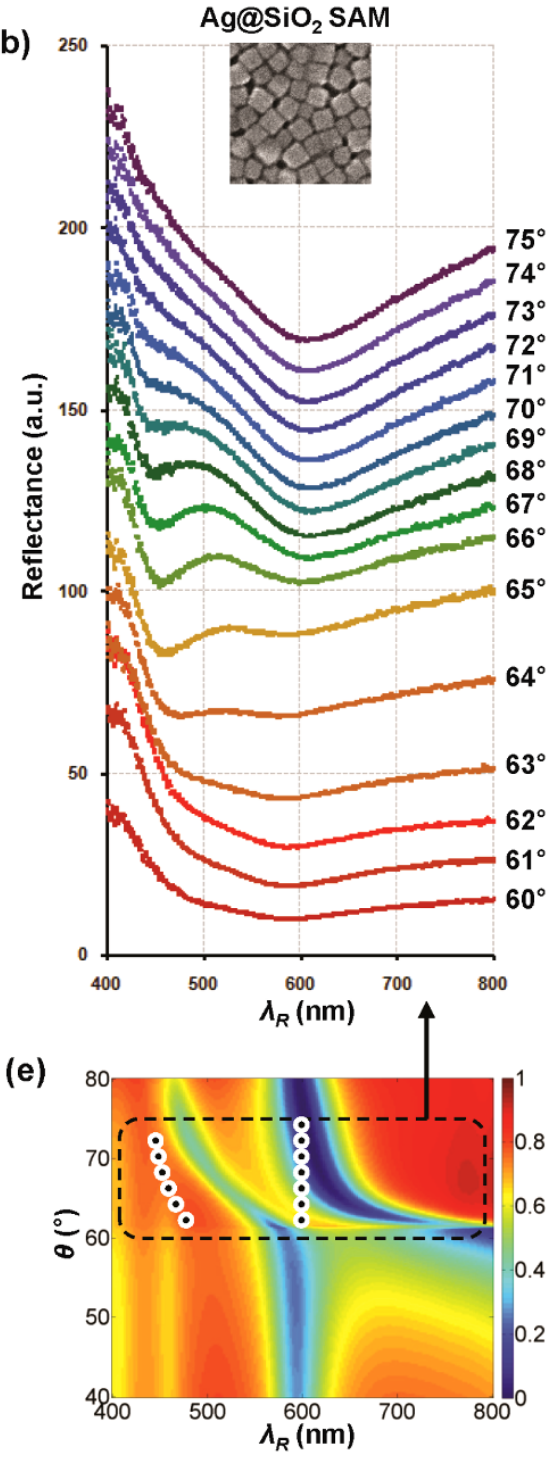

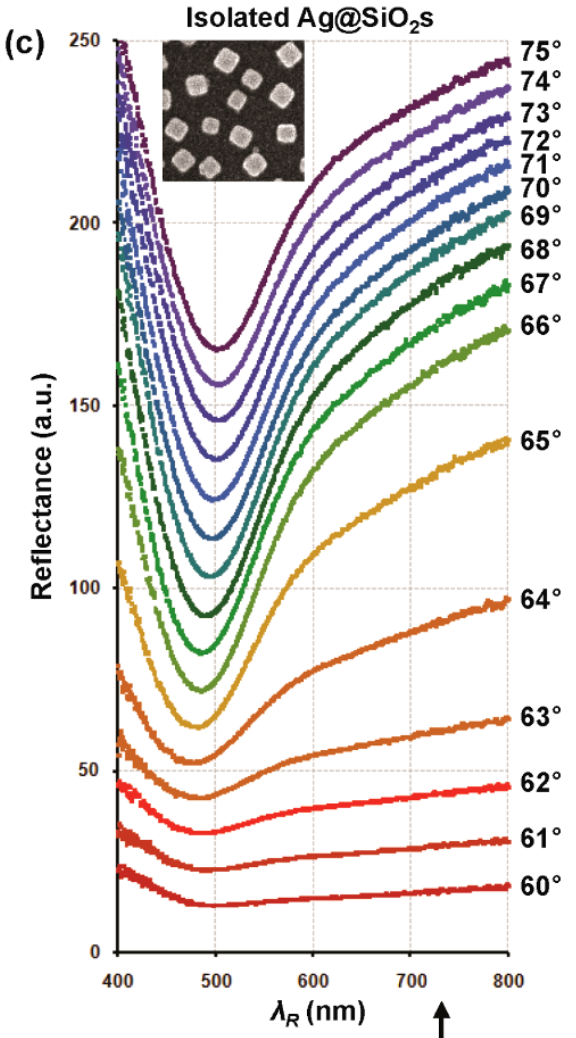

(f)

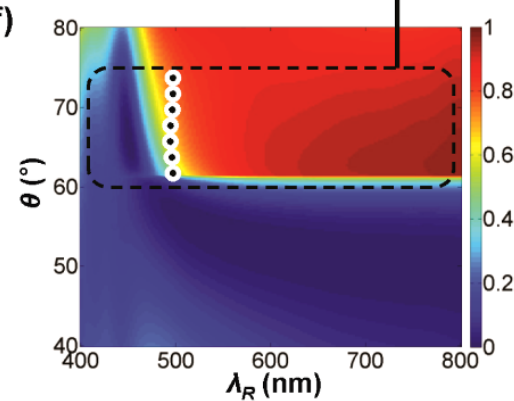

Figure $4 \mid$ Experimental and simulation $\theta-\lambda_{R}$ maps. (a)-(c) experimental and (d)-(f) calculated $\theta-\lambda_{R}$ maps for AgNC (43 nm) with shell thickness of (a) and (d) $0 \mathrm{~nm}$, (b) and (e) $3.5 \mathrm{~nm}$, and (c) and (f) $21.5 \mathrm{~nm}$. The positions of the dips on the experimental maps of (a)-(c) are marked with round black dots at the same positions on the simulation maps of (d)-(f). The whole regions in the experimental maps are denoted by dotted boxes on the simulation maps. 
index 1.00) as surrounding medium are shown in Fig. S5 of the Supplementary Information. When the spatial distribution of the particles is applied to the simulation condition, two bands become broad and the reflectance increases slightly while the overall tendency of $\theta-\lambda_{R}$ map remains the same. In particular, the blue-shift of the left band is bigger than that of the right band because the short wavelength of light can identify the variation of the distribution more accurately than the long wavelength. Consequentially, in the comprehensive analysis the experimental $\theta-\lambda_{R}$ map became more similar to the $\theta-\lambda_{R}$ map in Fig. 5(f) than that in Fig. 5(d)) through statistical process. The similarity between the experimental and calculated graphs can give a validity of $2 \mathrm{D}$ simulation at least in this case.

\section{Discussion}

We acquired the distinguishing $\theta-\lambda_{R}$ maps of the PSP-LSP coupling in an $\mathrm{AgNC} @ \mathrm{SiO}_{2}$ monolayer using 2D simulation to effectively process the enormous data. To verify the appropriateness of the $2 \mathrm{D}$ simulation results, representative $3 \mathrm{D}$ simulations were conducted. The angle $(\varphi)$ between the perpendicular plane ( $x z$-plane) to the monolayer and the orthogonally projected incident light on the monolayer ( $x y$-plane) was additionally considered in $3 \mathrm{D}$ simulations, as shown in Fig. S6(a) of the Supplementary Information. In the rigorous coupled wave analysis (RCWA), the accuracy of numerical results is proportional to the number of eigenmodes that were used for calculation, but it has trade-off relations with computation time $^{19-22}$. Therefore, we conducted 3D simulation with lower eigenmodes and resolution than those of $2 \mathrm{D}$ simulation in the limited region of wavelengths from $520 \mathrm{~nm}$ to $720 \mathrm{~nm}$ and incident angles from $55^{\circ}$ to $70^{\circ}$, which is depicted by black lines in Fig. S6(b). As results of $3 \mathrm{D}$ simulations, the shape and position of two hybrid bands on $\theta-\lambda_{R}$ map are similar to those of the $2 \mathrm{D}$ simulation result as in
Figs. S6(b)-(d), even though minute differences can be missed due to the relatively low number of eigenmodes. Therefore, the similarity between $2 \mathrm{D}$ and $3 \mathrm{D}$ calculations supports the reliability of the $2 \mathrm{D}$ simulation results.

The intermediate characteristics of the PSP-LSP coupling in QCML lead us to interesting subjects like band engineering and slow SPs. In QCML, the electromagnetic fields are changed between the intrinsic states of PSPs and LSPs as in Figs. 1(g)-(l). This means that SPs on the same structure can be minutely tuned by changing the excitation conditions. In particular, the lower energy band in Fig. 1(d) can be regarded as a new dispersion characteristic modified from that of PSPs in a conventional metal surface. In this band, the group velocity decreases due to the effects of LSPs. From our numerical results, the group velocity $\left(v_{\mathrm{g}}\right)$ of the SPs in our QCML can be lowered down to the less than one fourth times to that of SPs in a conventional metal layer. Therefore, it can be potentially applicable to design the energy bands in plasmonics or to generate the slow SPs.

In conclusion, we can hybridize two conspicuous dispersion bands with the PSP-LSP coupling in a QCML and concretely demonstrate their intermediate behaviors depending on structural parameters. Although a precise QCML structure with a constant gap of few nanometers cannot be realized yet with neither top-down nor bottom-up process in current technology, the intermediate characteristics between PSPs and LSPs on the proposed structure will clearly show the distinguishing property if an innovative process for the nanofabrication is developed in future. The intermediate characteristics have the advantage to simultaneously excite PSPs and LSPs in one structure: for examples, because PSP- and LSP-based bio sensors having different optimum conditions in sensitivity and resolution for target analytes ${ }^{46,47}$ can be complementally combined in one chip, the complex interactions of individual biomolecules and a bulk cell can

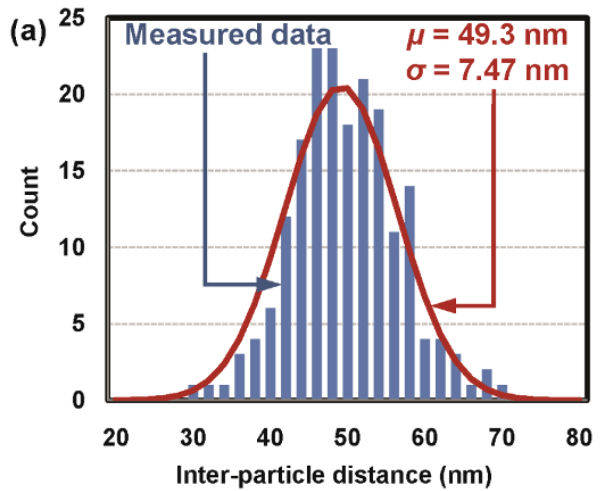

(d)

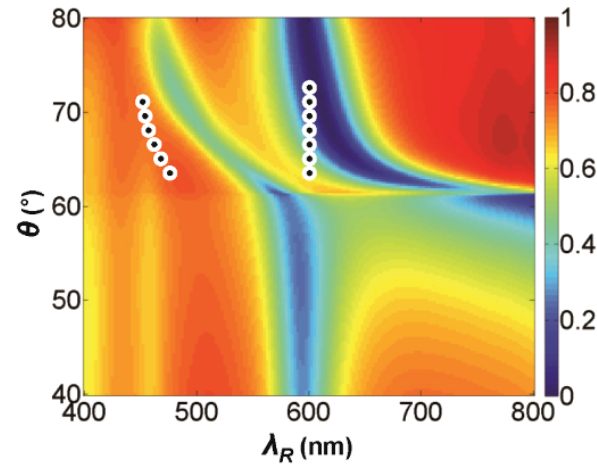

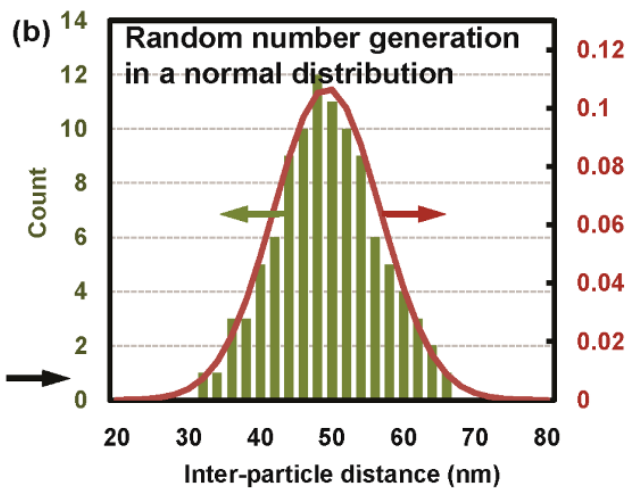

(e)

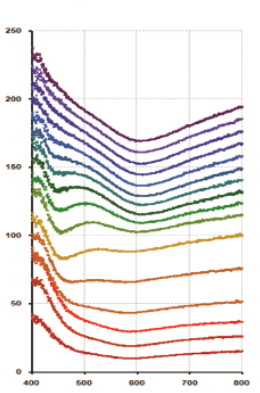

Normal distribution of inter-particle distances

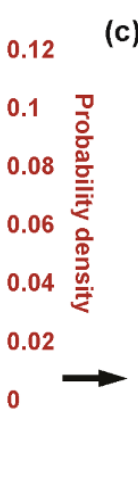

(f)

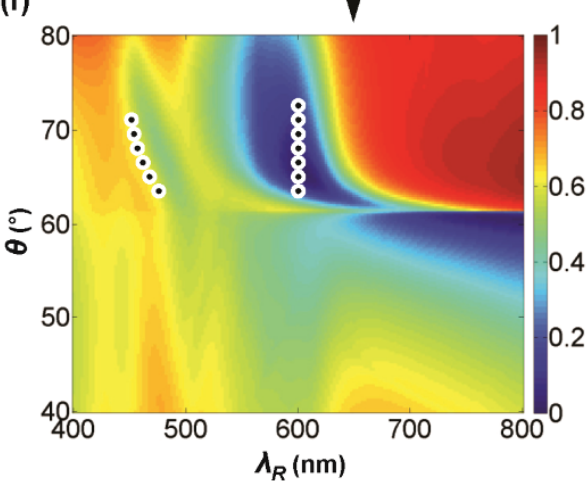

Figure 5 Statistical feedback of spatial distribution of $\mathrm{AgNC} @ S i O_{2}$ s to simulation. (a) Inter-particle distance distribution of AgNC@SiO ${ }_{2}$ in sample. The average inter-particle distance is $49.3 \mathrm{~nm}$ and standard deviation is $7.47 \mathrm{~nm}$. (b) Randomly generated distribution with normality. (c) Schematic diagram of simulation structure and condition for random distribution of inter-particle distances. One period includes one hundred of randomly distributed cubic units with normality. $P, d, t, \theta$, and $R$ are period, core diameter, shell thickness, incident angle, and reflectance, respectively. (d) Initial calculated, (e) experimental, and (f) recalculated $\theta-\lambda_{R}$ maps. The positions of the dips on the experimental map in (e) are marked with round black dots at the same positions on the simulation maps of $(\mathrm{d})$ and $(\mathrm{f})$. 
be detected by different states of SPs. The control on the energy bands and speed of SPs in a QCML can also provide the fundamental methods to research nanophotonic signal processing ${ }^{48,49}$. Furthermore, if it is combined with various flexible or nonlinear materials, it will potentially contribute to developing active nanophotonic devices because the structural and dielectric properties can be tun$a_{b l e}{ }^{50,51}$ to actively utilize the multiple bands and PSP-LSP transition in a QCML.

\section{Methods}

Simulations. For the calculation of electromagnetic fields and reflectance on the proposed structure, our own-built rigorous coupled wave analysis (RCWA) tool is used $^{19-22}$. The numerical method is firstly to divide overall geometry as an array of layers and to calculate the Fourier spectrum of each layer. Then, eigenmodes which can exist in those layers are obtained. Finally, coupling coefficients from the incident wave to eigenmodes in each layer are calculated by using the extended transfer matrix method (ETMM). By summing backwardly the propagating coupling coefficients for all the eigenmodes existing in the incident layer, we could obtain reflectance for a specific wavelength and incident angle. $\theta-\lambda_{R}$ map is calculated from the repeated process of RCWA calculation varying with the condition of incident angle and wavelength.

Preparation of AgNC@SiO 2 s. $50 \mathrm{mg} / \mathrm{mL}$ solution of silver nitrate $\left(\mathrm{AgNO}_{3}\right.$, SigmaAldrich), $25 \mathrm{mg} / \mathrm{mL}$ of poly(vinyl pyrrolidone) (PVP, Sigma-Aldrich), and $5 \mathrm{mM}$ solution of sodium hydrosulfide (NaHS, Sigma-Aldrich) in ethylene glycol (EG) were prepared. $8 \mathrm{~mL}$ of EG was heated at $150^{\circ} \mathrm{C}$ and stirred with a silicon coated magnetic bar for $1 \mathrm{~h}$ in a $20 \mathrm{~mL}$ glass vial. $0.05 \mathrm{~mL}$ of the sulfide solution, $1.8 \mathrm{~mL}$ of the PVP solution and $0.4 \mathrm{~mL}$ of the $\mathrm{AgNO}_{3}$ solution were sequentially injected into the heated EG solution. After 3-5 min of reaction time, the solution was quenched with cooling water. AgNCs were dispersed in ethanol after three times of rinsing processes of centrifugation, dilution, and sonication with acetone and ethanol. To wrap $\mathrm{SiO}_{2}$ shell around AgNC, 25\% solution of ammonia and 10\% solution of tetraethoxysilane (TEOS, Sigma-Aldrich) in ethanol were prepared. $7.6 \mathrm{~mL}$ ethanol was stirred in $20 \mathrm{~mL}$ glass vial and then $3 \mathrm{~mL}$ of the AgNC solution, $0.2 \mathrm{~mL}$ of the ammonia solution and $0.6 \mathrm{~mL}$ of the TEOS solution were sequentially injected into the ethanol in the vial. The mixed solution was stirred at room temperature for $12 \mathrm{~h}$. After reaction, excess reactants were washed away in the rinsing process.

Self-assembled monolayer of ${\mathrm{AgNC} @ S i \mathrm{O}_{2}}_{2}$. A glass substrate with the hydrophilic surface was immersed in a clean Petri dish filled with DI water for AgNC@SiO ${ }_{2}$ SAM. $\mathrm{AgNC} @ \mathrm{SiO}_{2} \mathrm{~S}$ were dispersed in 1-butanol and then were slowly dropped onto the water surface. Once the SAM was formed at the interface of air and water, it was settled on the glass substrate by natural drying.

Optical measurement. Surface morphologies of the samples were measured with a field emission scanning electron microscope (FE-SEM; JSM 6700F, JEOL) and a high resolution transmission electron microscope (HR-TEM; JEM-3010, JEOL). The samples were illuminated from the bottom by white light of halogen lamp with changing the incident angle from $40^{\circ}$ to $70^{\circ}$. Reflectance spectra were measured using a spectrometer (SM240, Spectral Products) at the same angle with that of incident light.

1. Ebbesen, T. W., Lezec, H. J., Chaemi, H. F., Thio, T. \& Wolff, P. A. Extraordinary optical transmission through sub-wavelength hole arrays. Nature 391, 667-669 (1998).

2. Pendry, J. B., Martin-Moreno, L. \& Garcia-Vidal, F. J. Mimicking surface plasmons with structured surfaces. Science 305, 847-848 (2004).

3. Seo, M. A. et al. Terahertz field enhancement by a metallic nano slit operating beyond the skin-depth limit. Nat. Photonics 3, 152-156 (2009).

4. Raether, H. R. Surface Plasmons on Smooth and Rough Surfaces and on Gratings (Springer, Berlin, 1988).

5. Maier, S. Plasmonics: Fundamentals and Applications (Springer, New York, 2007).

6. Brongersma, M. L. \& Kik, P. G. Surface Plasmon Nanophotonics (Springer, Dordrecht, 2007).

7. Kreibig, U. \& Vollmer, M. Optical Properties of Metal Clusters (Springer, Berlin, 1995).

8. Kelly, K. L., Coronado, E., Zhao, L. L. \& Schatz, G. C. The optical properties of metal nanoparticles: the influence of size, shape, and dielectric environment. J. Phys. Chem. B 107, 668-677 (2003).

9. Lal, S., Link, S. \& Halas, N. J. Nano-optics from sensing to waveguiding. Nat. Photonics 1, 641-648 (2007)

10. Pelton, M., Aizpurua, J. \& Bryant, G. Metal-nanoparticle plasmonics. Laser \& Photon. Rev. 2, 136-159 (2008).

11. Cesario, J., Quidant, R., Badenes, G. \& Enoch, S. Electromagnetic coupling between a metal nanoparticle grating and a metallic surface. Opt. Lett. 30, 3404-3406 (2005)

12. Ghoshal, A. \& Kik, P. G. Theory and simulation of surface plasmon excitation using resonant metal nanoparticle arrays. J. Appl. Phys. 103, 113111 (2008).
13. Chu, Y., Banaee, M. G. \& Crozier, K. B. Double-resonance plasmon substrates for surface-enhanced raman scattering with enhancement at excitation and stokes frequencies. ACS Nano 4, 2804-2810 (2010).

14. Yu, F. et al. Simultaneous excitation of propagating and localized surface plasmon resonance in nanoporous gold membranes. Anal. Chem. 78, 7346-7350 (2006).

15. Hu, C., Liu, L., Zhao, Z., Chen, X. \& Luo, X. Mixed plasmons coupling for expanding the bandwidth of near-perfect absorption at visible frequencies. Opt. Express 17, 16745-16749 (2009)

16. Schwind, M., Kasemo, B. \& Zorić, I. Localized and propagating plasmons in metal films with nanoholes. Nano Lett. 13, 1743-1750 (2013).

17. Collier, C. P., Saykally, R. J., Shiang, J. J., Henrichs, S. E. \& Heath, J. R. Reversible tuning of silver quantum dot monolayers through the metal-insulator transition. Science 277, 1978-1981 (1997).

18. Tao, A., Sinsermsuksakul, P. \& Yang, P. Tunable plasmonic lattices of silver nanocrystals. Nat. Nanotechnol. 2, 435-440 (2007).

19. Moharam, M. G., Grann, E. B. \& Pommet, D. A. Formulation for stable and efficient implementation of the rigorous coupled-wave analysis of binary gratings. J. Opt. Soc. Am. A 12, 1068-1076 (1995).

20. Lalanne, P. \& Silberstein, E. Fourier-modal methods applied to waveguide computational problems. Opt. Lett. 25, 1092-1094 (2000).

21. Kim, H., Lee, I.-M. \& Lee, B. Extended scattering-matrix method for efficient full parallel implementation of rigorous coupled-wave analysis. J. Opt. Soc. Am. A 24, 2313-2327 (2007).

22. Kim, H., Park, J. \& Lee, B. Fourier Modal Method and Its Applications in Computational Nanophotonics (CRC Press, New York, 2012).

23. Huang, W.-P. Coupled-mode theory for optical waveguides: an overview. J. Opt. Soc. Am. A 11, 963-983 (1994).

24. Yariv, A., Xu, Y., Lee, R. K. \& Scherer, A. Coupled-resonator optical waveguide: a proposal and analysis. Opt. Lett. 24, 711-713 (1999).

25. Prodan, E., Radloff, C., Halas, N. J. \& Nordlander, P. A hybridization model for the plasmon response of complex nanostructures. Science 302, 419-422 (2003).

26. Oulton, R. F., Sorger, V. J., Genov, D. A., Pile, D. F. P. \& Zhang, X. A hybrid plasmonic waveguide for subwavelength confinement and long-range propagation. Nat. Photonics 2, 496-500 (2008).

27. Maier, S. A., Kik, P. G. \& Atwater, H. A. Observation of coupled plasmonpolariton modes in Au nanoparticle chain waveguides of different lengths: estimation of waveguide loss. Appl. Phys. Lett. 81, 1714-1716 (2002).

28. Maier, S. A. et al. Local detection of electromagnetic energy transport below the diffraction limit in metal nanoparticle plasmon waveguides. Nat. Mater. 2, 229-232 (2003)

29. B. Lamprecht, G. et al. Metal nanoparticle gratings: influence of dipolar particle interaction on the plasmon resonance. Phys. Rev. Lett. 84, 4721-4724 (2000).

30. Bohren, C. F. \& Huffman, D. R. Absorption and Scattering of Light by Small Particles (Wiley, New York, 1983).

31. Kreibig, U. \& Genzel, L. Optical absorption of small metallic particles. Surf. Sci. 156, 678-700 (1985).

32. Lu, Y. \& Lal, A. Vacuum-free self-powered parallel electron lithography with sub35-nm resolution. Nano Lett. 10, 2197-2201 (2010).

33. Pan, L. et al. Maskless plasmonic lithography at $22 \mathrm{~nm}$ resolution. Sci. Rep. 1, 175 (2011).

34. Black, C. T. et al. Polymer self assembly in semiconductor microelectronics. IBM J. Res. Dev. 51, 605-633 (2007).

35. Tada, Y. et al. Directed self-assembly of POSS containing block copolymer on lithographically defined chemical template with morphology control by solvent vapor. Macromolecules 45, 292-304 (2012).

36. Buining, P. A., Humbel, B. M., Philipse, A. P. \& Verkleij, A. J. Preparation of functional silane-stabilized gold colloids in the (sub)nanometer size range. Langmuir 13, 3921-3926 (1997).

37. Ostrowski, A. D. et al. Controlled synthesis and single-particle imaging of bright, sub-10 nm lanthanide-doped upconverting nanocrystals. ACS Nano 6, 2686-2692 (2012).

38. Im, S. H., Lee, Y. T., Wiley, B. \& Xia, Y. Large-scale synthesis of silver nanocubes: the role of $\mathrm{HCl}$ in promoting cube perfection and monodispersity. Angew. Chem. Int. Ed. 44, 2154-2157 (2005)

39. Siekkinen, A. R., McLellan, J. M., Chen, J. \& Xia, Y. Rapid synthesis of small silver nanocubes by mediating polyol reduction with a trace amount of sodium sulfide or sodium hydrosulfide. Chem. Phys. Lett. 432, 491-496 (2006).

40. Liz-Marzán, L. M., Giersig, M. \& Mulvaney, P. Synthesis of nanosized gold-silica core-shell particles. Langmuir 12, 4329-4335 (1996)

41. Graf, C., Vossen, D. L. J., Imhof, A. \& van Blaaderen, A. A general method to coat colloidal particles with silica. Langmuir 19, 6693-6700 (2003).

42. Reincke, F., Hickey, S. G., Kegel, W. K. \& Vanmaekelbergh, D. Spontaneous assembly of a monolayer of charged gold nanocrystals at the water/oil interface. Angew. Chem. Int. Ed. 43, 458-462 (2004).

43. Moon, G. D. et al. Assembled monolayers of hydrophilic particles on water surfaces. ACS Nano 5, 8600-8612 (2011).

44. Wen, T. \& Majetich, S. A. Ultra-large-area self-assembled monolayers of nanoparticles. ACS Nano 5, 8868-8876 (2011).

45. Gao, B., Arya, G. \& Tao, A. R. Self-orienting nanocubes for the assembly of plasmonic nanojunctions. Nat. Nanotechnol. 7, 433-437 (2012). 
46. Svedendahl, M., Chen, S., Dmitriev, A. \& Käll, M. Refractometric sensing using propagating versus localized surface plasmons: a direct comparison. Nano Lett. 9, 4428-4433 (2009).

47. Roh, S., Chung, T. \& Lee, B. Overview of the characteristics of micro- and nanostructured surface plasmon resonance sensors. Sensors 11, 1565-1588 (2011).

48. Vlasov, Y. A., O’Boyle, M., Hamann, H. F. \& McNab, S. J. Active control of slow light on a chip with photonic crystal waveguides. Nature 438, 65-69 (2005).

49. Fedyanin, D. Yu. \& Arsenin, A. V. Stored light in a plasmonic nanocavity based on extremely-small-energy-velocity modes. Phot. Nano. Fund. Appl. 8, 264-272 (2010).

50. Pryce, I. M., Aydin, K., Kelaita, Y. A., Briggs, R. M. \& Atwater, H. A. Highly strained compliant optical metamaterials with large frequency tunability. Nano Lett. 10, 4222-4227 (2010).

51. Kauranen, M. \& Zayats, A. V. Nonlinear plasmonics. Nat. Photonics 6, 737-748 (2012)

\section{Acknowledgments}

This work was supported by the National Research Foundation of Korea (NRF) grant funded by the Korean government (MSIP) through the Creative Research Initiatives

Program (Active Plasmonics Application Systems).

\section{Author contributions}

H.Y. conceived the ideas and executed the experiments. H.Y. and S.-Y.L. performed the simulations. H.Y., I.-M.L., S.-Y.L. and K.-Y.K. analyzed the results and prepared the manuscript. B.L. supervised the project. All authors discussed the results and commented on the manuscript.

\section{Additional information}

Supplementary information accompanies this paper at http://www.nature.com/ scientificreports

Competing financial interests: The authors declare no competing financial interests.

How to cite this article: Yun, H., Lee, I.-M., Lee, S.-Y., Kim, K.-Y. \& Lee, B. Intermediate plasmonic characteristics in a quasi-continuous metallic monolayer. Sci. Rep. 4, 3696; DOI:10.1038/srep03696 (2014).

\section{NonCommercial-ShareAlike 3.0 Unported license. To view a copy of this license,} visit http://creativecommons.org/licenses/by-nc-sa/3.0 